\title{
KURZBESPRECHUNGEN
}

Richard Collins, James Curran, Nicholas Garnbam u.a. (Hrsg.): Media, Culture and Society. A Critical Reader. London: Sage 1986. 345 Seiten.

Zur Übernahme der Zeitschrift "Media, Culture and Society " hat der Sage Verlag unter diesem Zeitschriftentitel die wichtigsten Beiträge der früheren Jahrgänge hier in einem Buch noch einmal zugänglich gemacht. Dabei werden die Nachdrucke in drei Gruppen gegliedert. 1. "Approaches to cultural theory " mit Beiträgen von Nicholas Garnham, Stuart Hall, John Corner und Michele Mattelart. 2. "Intellectuals and cultural production « mit Beiträgen von Philip Schlesinger, Philip Elliott, Nicholas Garnham und Raymond Williams, Pierre Bourdieu, Paul Dimaggio.

Entsprechend der Zeitschrift selbst kommen die Beiträge dieses Bandes aus dem Umfeld britischer (und kritischer!) Kommunikationsforschung, bei der die Massenmedien eben nur als ein Aspekt des Gesamtbereiches Kultur gesehen werden. Auf diesem Hintergrund sind dann auch die Beiträge der dritten Beitragsgruppe dieses Bandes unter dem Titel "British Broadcasting and the public sphere" zu sehen.

Diese Aufsatzsammiung wird in Zukunft ein Standardwerk für jeden sein, der sich kritisch mit der Kommunikation in Kultur und Gesellschaft auseinandersetzt.

F.-J.E.

Handbuch des Wettbewerbsrechts, hrsg. von Wolfgang Gloy. München 1986. LV III und 1197 SS. - Nachtrag UWG-Novelle 1986. München 1987.59 SS.

Nicht nur für die Medienkaufleute, Marketingfachleute und Werbeagenturen, sondern auch für viele Journalisten ist das Wettbewerbsrecht in den letzten Jahren bedeutsam geworden. Deshalb hat der Herausgeber in Gemeinschaft mit siebzehn Autoren - alle Richter oder Rechtsanwälte mit langjähriger Praxis auf dem Gebiet des Wettbewerbsrechts - den Versuch unternommen, ein auf die Bedürfnisse der Praxis zugeschnittenes Nachschlagewerk zu schaffen. Ausgehend von einem weiten Verständnis des Begriffs des Wettbewerbsrechts stellt das Handbuch nicht nur das Recht gegen den unlauteren Wettbewerb mit seinen zahlreichen Nebengesetzen dar, sondern auch das Recht gegen Wettbewerbsbeschränkungen - das Kartellrecht jedoch nur, soweit es für die Beurteilung der werblichen oder vertrieblichen Aktivitäten eines Unternehmens von Bedeutung ist.

Das Handbuch gliedert sich in drei Hauptteile. In einem ersten Teil werden die allgemeinen wettbewerbsrechtlichen Grundbegriffe und die neueren Entwicklungen dieses Rechtsgebietes im internationalen und im EGBereich dargestellt. Der zweite Teil befaßt sich mit den unzulässigen Wettbewerbshandlungen. Dieser ausführlichste Teil des Handbuchs (über $650 \mathrm{SS}$ ) befaßt sich mit Beschränkungen und Behinderungen im Wettbewerb, Wettbewerbswidrigkeit durch Rechtsbruch, unlautere Handlungen gegenüber Abnehmern, besondere Verkaufsveranstaltungen und dem Schutz geschäftlicher Kennzeichnungen und Warenzeichen. Im dritten Teil ist das Verfahrensrecht zentraler Themenkreis, in dem die Abmahnung, das Verfügungsverfahren und die Hauptsacheklage umfassend dargestellt werden.

Über das Wettbewerbs- und Kartellrecht hinausgreifend dargestellt werden besonders aktuelle Themen. Aus publizistischem Blickwinkel besonders interessant sind hier im Kapitel "Neue Medien« die Paragraphen 27 und 28, die sich mit Bildschirmtext und Satellitenwerbung befassen. Die durch das Gesetz zur Änderung wirtschafts-, verbraucher-, arbeitsund sozialrechtlicher Vorschriften vom 25.7.1986 erfolgte Novellierung des UWG ist Gegenstand eines Nachtrages.

Ein ausführliches Sachverzeichnis erschließt - neben der detaillierten Gliederung - das Handbuch; hilfreich die Textpassage. Die Absicht von Herausgeber und Autoren, eine umfassende und dennoch dem Laien verständliche Darstellung zu erreichen, ist voll gelungen. Dieser Band gehört in jede Redaktionsbibliothek. 
"Lebrer belfen Lebrern e.V., Neuss": "Kind 8 Buch - Literatur für/über Kinder«. Neuss 1987. 93 S.

Diese kleine Bibliographie verdankt ihre Entstehung einer bemerkenswerten Initiative. Der Neusser Verein "Lehrer helfen Lehrern « ist von Lehrern gegründet worden, die ihren arbeitslosen Kollegen helfen wollen, in pädagogischen Arbeitsfeldern außerhalb des normalen Schulunterrichts tätig zu werden. Eine Frucht dieser Bemühungen ist der vorliegende Versuch, aus der Fülle der Neuerscheinungen auf dem Sektor der Bücher für und über Kinder eine sinnvolle Auswahl unter kindgerechten und pädagogisch geleiteten Aspekten zu treffen. Herausgekommen ist dabei eine bibliografisch sauber gearbeitete Handreichung, die man sich in die Hand vieler Eltern und Erzieher wünscht. Die Bücher sind geschickt nach Leitbegriffen geordnet, so daß man rasch das Passende findet. Fast $\mathrm{zu}$ jedem Buch ist außerdem eine hilfreiche Kurzangabe beigefügt, die auf den Inhalt eingeht und die Altersgruppe angibt, für die die Bücher gedacht sind.

B.M.

Nowotny, Burkhard: Rundfunk bürgernah. Regionalisierung, lokale Sender und Privatfunk in Großbritannien. Hameln 1982.240 SS.

Unserer Zeit, besser noch: der medienpolitisch ermöglichten Entwicklung, voraus war die für bundesrepublikanische Verhältnisse frühzeitige Publikation britischer Entwicklungen auf den Gebieten der Regionalisierung, Lokalisierung und Privatisierung des Rundfunks. Weil erst in jüngster Zeit bei uns Realität ist, was in Großbritannien bereits historisch genannt werden kann, sei auf dieses informative Buch verwiesen, das - frei von kulturpessimistischen Suaden und medieneuphorischen Pirouetten - nicht nur die geschichtliche Entwicklung schildert und differenziert, sondern auch - basierend auf britischem Quellenmaterial - Ergebnisse der Hörerforschung einbezieht. Die Übertragbarkeit der Verhältnisse in Großbritannien auf die Bundesrepublik mag zwar nicht immer direkt einleuchten, doch können zahlreiche Erfahrungen hier nutzbringend *übersetzt « werden. Vielleicht gelingt ja sogar eine Übertragung des britischen Rundfunk-Grundsatzes: „The most important person in broadcasting is the listener «. Lesenswert für alle, die mit Lokalfunk in Berührung kommen und - für den Verlag - empfehlenswert, dieses Buch bis in die jüngste Gegenwart fortzuschreiben.

G.D.

Presse- und Informationsamt der Bundesregierung (Hrsg.): Kommunikationspolitische und kommunikationswissenschaftliche Forschungsprojekte der Bundesregierung (1978 1985). Eine Übersicht über wichtige Ergebnisse (2 Teile). Bonn 1986. 808 SS.

Nach 1974 und 1978 zum dritten Mal im Jahr 1986 legt das Presse- und Informationsamt der Bundesregierung eine Übersicht über die von der Bundesregierung zu bestimmten Fragestellungen der Medienstruktur und Kommunikationspolitik veranlaßten Forschungsberichte vor. Diese 72 Forschungsberichte spiegeln nicht das Interesse der Regierung wider, umfassend wissenschaftlich fundierte Medienforschung zu betreiben, sondern man will zu jeweils aktuellen Problemen der medienpolitischen Diskussion Antworten auf Fragen erhalten, zu denen keine oder nur unzureichende Darstellungen vorliegen. Während die Zahl der Projekte gegenüber früher zugenommen hat, flachte der Anstieg der hierfür zur Verfügung stehenden Mittel ab; Großprojekte waren die Ausnahme. Stärker als früher tritt der subsidiäre Charakter der Untersuchungsaufträge gegenüber der von der Publizistik- und Kommunikationswissenschaft selber initiierten Forschung zutage.

Gegenüber den früheren Berichtszeiträumen haben sich neue Schwerpunkte bei der Themenstellung der Forschungsschwerpunkte ergeben. Während in vergangenen Zeiten Studien zur Situation der im Medienbereich Tätigen, zur Struktur der Presse und zu rechtlichen Fragen überwogen, schoben sich nun die Neuen Medien « in den Vordergrund. Allein 23 Studien betreffen die (vermuteten) sozialen Auswirkungen der Informations-und Kommunikationstechniken und die potentiellen Folgen für die existente Medienstruktur.

Die einzelnen Projekte bilden die Forschungsfelder:

\section{Bestehendes Medienangebot}

\subsection{Kommunikationsforschung}

1.2. Medienstruktur und-angebot 
1.3. Medieninhalte

1.4. Nutzung und Wirkung

1.5. Wirtschaft und Werbung

1.6. Rechtliche und politische Fragen

2. "Neue Medien*

2.1. Akzeptanz und voraussichtliche Entwicklung

2.2. Auswirkungen auf die bestehende Medienstruktur

2.3. Gesellschaftliche Folgen

3. Bibliographie und Dokumentation im Medienbereich

Neben den einmal mehr oder auch einmal weniger ausführlichen Zusammenfassungen der Untersuchungsergebnisse werden - wenn die Gutachten veröffentlicht werden - die bibliographischen Daten angegeben. Die beiden Bände schließen mit einer Dokumentation der von 1971 bis 1978 von der Bundesregierung geförderten Forschungsprojekte und einem Namens- und Sachregister.

G.D.

George Wedell (Hrgb.): Making Broadcasting useful: The African Experience. The Development of Radio and Television in Africa in the 1980s. Manchester: Manchester University Press 1986. 306 Seiten.

Dieses Buch ist keine Einzelstudie, wie der Untertitel nahelegen könnte. Es handelt sich um einen Sammelband mit 25 verschiedenen Beiträgen zum gestellten Thema. Bemerkenswert ist dabei vor allem, daß die meisten dieser Texte von afrikanischen Autoren stammen. Hier ist - wohl zum ersten Mal - der Versuch einer umfassenden Darstellung der Situation und der Probleme des Rundfunks und seiner Planung in Afrika durch Beteiligte selbst gemacht.

Dabei werden die Einzelbeiträge - wohl alle nur aus dem englischsprachigen Afrika - in drei Gruppen gegliedert: 1. Curent issues, 2. some country studies, und 3. "An agenda for the future $*$. Generell geht es bei allen Beiträgen nicht so sehr um die Technik, die 'hardwa- re', sondern mehr um grundsätzliche Fragen. Dabei wird den Autoren in der Einleitung deswegen Kompetenz bescheinigt, weil sie in Politik, Administration, Planung oder Forschung aktiv seien (S. XI). Gerade hier aber möchte man gelegentlich ein Fragezeichen machen; denn die Erfahrung zeigt, daß manches Mal gerade Politiker und Verwaltung deswegen wirkliche Kommunikation vor allem für ländliche Bevölkerung be- oder verhindern, weil sie eben nicht wissen (wollen?), wovon sie bei ihrer politischen und städtisch bzw. westlich orientierten Sicht reden. Die Zugehörigkeit zu Politik und Verwaltung als solche jedenfalls ist oft noch kein genügender Grund für eine qualifizierte Aussage.

Die Beiträge des Sammelbandes insgesamt decken eine weite Bandbreite des Themas. Sie reichen von mehr beschreibenden Darstellungen zu kritischen Analysen und Forderungen. Hierher gehört etwa der Gebrauch verschiedener einheimischer Sprachen im Funk (Paul Ansah). Die Notwendigkeit einer fachlich qualifizierten Ausbildung wird betont, wobei man bei der Aufzählung der Kursforderungen u.a. das so entscheidende 'Scriptwriting' vermißt (S. 68) ...

Ein Teil der Beiträge dieses Buches stammt von einem Symposium, welches das herausgebende 'European Institute for the Media' an der Universität von Manchester veranstaltet hat. So schließt denn das Werk auch mit 15 Empfehlungen, die zu weiterem Nachdenken und zur Planung anregen sollen. Sie fordern u.a. eine neue, nüchterne Beurteilung der Möglichkeiten des Funks vor allem im Blick auf die tatsächlichen lokalen und nationalen Notwendigkeiten. Der Funk muß den wirklichen Bedürfnissen des Hörers in seiner Kultur dienen. Regelmäßige Hörerforschung ist dafür ebenso notwendig wie die Förderung einheimischer Kunst und eigener Produktionen und die Heranbildung eigenen, fachlich qualifizierten Personals. Die Rolle des Rundfunks für Entwicklung sollte sowohl vom Programm als auch von den technischen Möglichkeiten her besser durchdacht und ernster angefaßt werden.

K.A. 


\section{ZEITSCHRIFTENHINWEISE}

\section{Allgemeine Publizistik}

Baragli, E.: Orientamenti per i futuri sacerdoti circa gli strumenti della comunicazione sociale. In: La Civilta Cattolica (Roma) Quaderno 3277, 26-39.

Eilers, Franz-Josef: Common Witness and Social Communication. In: Verbum (Roma) 1/87, 70-75.

Foley, John F.: Das Wort Gottes in den Medien. (Ansprache v. Erzbischof John F. Foley vor der Katholischen Weltunion der Presse am 13.03.86 in Madrid) In: Anregung (Nettetal) 3/87, 146-147.

Hengst, Heinz: "An die wirklichen Probleme der Gesellschaft kommt man mit Medienpädagogik nicht heran. « Interview mit der Herder Korrespondenz. In: Herder Korrespondenz (Freiburg) 4/87, 166-172.

Kodalle, Klaus M.: Versprachlichung des Sakralen? Zur relig.-phil. Auseinandersetzung mit Jürgen Habermas' "Theorie des kommunikativen Handelns « In: Allgemeine Zeitschrift für Philosophie (Stuttgart) 1/87, 39-66.

Meiden, Anne von der: Kommunikation, $\mathrm{Me}-$ dien und Moral. In: Zeitschrift für evangelische Ethik (Gütersloh) 2/87, 190-209.

Merkert, Rainald: Der neue Ruf nach Medienforschung. In: Stimmen der Zeit (Freiburg) 12/86, 855-857.

\section{Presse}

Biener, Hansjörg: Die »Zeitschrift für Protestantismus und Kirche « (1838-1876) in ihrer Entwicklung. In: Zeitschrift für bayrische Kirchengeschichte (Nürnberg) 1986, 113-158.

Buddenbaum, Judith M.: An Analysis of Religion. New Coverage In Three Major Newspapers. In: Journalism Quarterly (Columbia) 3/86, 600-606.

Kort, Wesley A.: Literatur und Theologie. In: Stimmen der Zeit (Freiburg) 2/87, 93-104 .

Schmidt-Sommer, Irmgard: Bibel und Gegenwartsliteratur. In: Erbe und Auftrag (Beuron) 2/87, 149-150.
Schrey, H.-H.: Christentum und Sozialismus. Ein Rückblick auf die Literatur der siebziger Jahre. In: Theologische Rundschau (Tübingen) $4 / 86,372-403$.

\section{Hörfunk/Fernsehen/Film}

Boventer, Hermann: Wir leben in Fernsehzeiten. "No Sense of Place«. In: Internationale Katholische Zeitschrift (Paderborn) 2/87, 178187.

Brownson, William C.: Radio Preaching. In: Reformed Review (Holland. Mich.) 1/86, 56.

Bundschub, Thomas: Filmkunst und Spiritualität. In: Wort und Antwort (Mainz) 6/86, 184.

Hoffmann, Berward: Erwachsenenkatechismus im Fernsehen. In: Katechetische Blätter (München) 2/87, 149.

Hornauer, Uwe: Weltuntergang im Kino. In: Evangelische Kommentare (Stuttgart) 2/87, 107.

Muth, Ludwig: Müssen wir das Fernsehen fürchten? In: Stimmen der Zeit (Freiburg) 3/87, 159-170.

Pablowsky, Peter: Das Fernsehen und die Orden. In: Ordensnachrichten (Wien) 1/87, 3638.

Schnelle, Josef: Wenn die Angst am größten ist... Bemerkungen zu Angst und Schrecken im Kino. In: Internationale Katholische Zeitschrift (Rodenkirchen) 1/87, 83-90.

Twaroch, Paul: Rundfunk und Orden. In: Ordensnachrichten (Wien) 1/87, 32-35.

\section{Neue Medien}

Kästner, Walter J.: Video in der pastoralen Jugendarbeit. In: Anzeiger (Freiburg) 2/87, 4850.

Röller, Dirk: Die Angst des Lehrers vor dem Computer (Zur Veränderung der Wirklichkeit durch neue Kommunikationsmedien und Computer aus der Sicht evangelischer Erzieher). In: Evangelische Kommentare (Stuttgart) 4/87, 200-203. 